\title{
Impact of 4 Lactobacillus plantarum capsular polysaccharide clusters on surface glycan composition and host cell signaling
}

Daniela M Remus ${ }^{1,2,3}$, Richard van Kranenburg ${ }^{4}$, Iris I van Swam ${ }^{1,2}$, Nico Taverne ${ }^{1,5}$, Roger S Bongers ${ }^{1,2}$, Michiel Wels ${ }^{1,2}$, Jerry M Wells ${ }^{1,5}$, Peter A Bron ${ }^{1,2,6}$ and Michiel Kleerebezem ${ }^{1,2,5^{*}}$

\begin{abstract}
Background: Bacterial cell surface-associated polysaccharides are involved in the interactions of bacteria with their environment and play an important role in the communication between pathogenic bacteria and their host organisms. Cell surface polysaccharides of probiotic species are far less well described. Therefore, improved knowledge on these molecules is potentially of great importance to understand the strain-specific and proposed beneficial modes of probiotic action.

Results: The Lactobacillus plantarum WCFS1 genome encodes 4 clusters of genes that are associated with surface polysaccharide production. Two of these clusters appear to encode all functions required for capsular polysaccharide formation (cps2A-J and cps4A-J), while the remaining clusters are predicted to lack genes encoding chain-length control functions and a priming glycosyl-transferase (cpsiA-I and cps3A-S). We constructed L. plantarum WCFS1 gene deletion mutants that lack individual ( $\Delta$ cps $1 A-I, \Delta c p s 2 A-J, \Delta c p s 3 A-J$ and $\Delta$ cps $4 A-J)$ or combinations of cps clusters ( $\Delta$ cps $1 A-3 \mathrm{~J}$ and $\Delta \operatorname{cps} 1 A-31, \Delta \operatorname{cps} 4 A-\Omega)$ and assessed the genome wide impact of these mutations by transcriptome analysis. The cps cluster deletions influenced the expression of variable gene sets in the individual cps cluster mutants, but also considerable numbers of up- and down-regulated genes were shared between mutants in cps cluster 1 and 2, as well as between mutant in cps clusters 3 and 4. Additionally, the composition of overall cell surface polysaccharide fractions was altered in each mutant strain, implying that despite the apparent incompleteness of cps $1 A-I$ and cps3A-J, all clusters are active and functional in L. plantarum. The $\triangle \operatorname{cps} 1 A-/$ strain produced surface polysaccharides in equal amounts as compared to the wild-type strain, while the polysaccharides were characterized by a reduced molar mass and the lack of rhamnose. The mutants that lacked functional copies of cps $2 A-J, c p s 3 A-J$ or cps $4 A-J$ produced decreased levels of surface polysaccharides, whereas the molar mass and the composition of polysaccharides was not affected by these cluster mutations. In the quadruple mutant, the amount of surface polysaccharides was strongly reduced. The impact of the cps cluster mutations on toll-like receptor (TLR)-mediated human nuclear factor (NF)-KB activation in host cells was evaluated using a TLR2 reporter cell line. In comparison to a L. plantarum wild-type derivative, TLR2 activation remained unaffected by the $\triangle \operatorname{cps} 1 A-I$ and $\Delta c p s 3 A-J$ mutants but appeared slightly increased after stimulation with the $\Delta \operatorname{cps} 2 A-J$ and $\Delta c p s 4 A-J$ mutants, while the $\Delta c p s 1 A-3 J$ and $\Delta \operatorname{cps} 1 A-3 J, \Delta c p s 4 A-J$ mutants elicited the strongest responses and clearly displayed enhanced TLR2 signaling.

(Continued on next page)
\end{abstract}

\footnotetext{
* Correspondence: Michiel.Kleerebezem@nizo.com

${ }^{1}$ TI Food \& Nutrition, Nieuwe Kanaal 9A, 6709 PA Wageningen,

The Netherlands

${ }^{2} \mathrm{NIZO}$ food research, Kernhemseweg 2,6718 ZB Ede, The Netherlands

Full list of author information is available at the end of the article
} 
(Continued from previous page)

Conclusions: Our study reveals that modulation of surface glycan characteristics in L. plantarum highlights the role of these molecules in shielding of cell envelope embedded host receptor ligands. Although the apparently complete cps clusters ( $c p s 2 A-J$ and $c p s 4 A-J)$ contributed individually to this shielding, the removal of all cps clusters led to the strongest signaling enhancement. Our findings provide new insights into cell surface glycan biosynthesis in L. plantarum, which bears relevance in the context of host-cell signaling by probiotic bacteria.

Keywords: Lactobacillus plantarum, Probiotic, Surface polysaccharides, Host cell signaling, TLR2 activation

\section{Background}

The Gram-positive bacterial cell envelope is a multilayered structure, which is mainly composed of peptidoglycan with embedded teichoic acids, proteins, and polysaccharides and which is essential to maintain cellular integrity and shape [1]. The molecules of the cell envelope collectively decorate the bacterial surface in a strain- and species-specific manner and facilitate important bacterial processes such as stress- and environmental-adaptation, surface colonization, and adhesion [2].

Lactic acid bacteria (LAB) are Gram-positive bacteria that can be encountered in a wide range of environmental niches, including a range of dairy and other food-raw material fermentations as well as the gastrointestinal (GI) tract of humans and animals. LAB have a clear industrial relevance, based on their role in food fermentation whereby they contribute to the preservation of raw-materials in foods, but also to the product's flavor and texture [3]. Additionally, specific LAB are marketed as health-promoting organisms or probiotics [4]. Most $\mathrm{LAB}$ are able to synthesize extracellular polysaccharides, many of which consist of heteropolysaccharides built up from regular repeating oligosaccharide units that commonly contain the monosaccharides galactose, glucose, and in several cases also encompass rhamnose, $N$-acetylglucosamine, $N$-acetyl-galactosamine, mannose, and non-carbohydrate substitutions [5-8]. The repeating units are synthesized in the cytoplasm and assembled on the lipid carrier undecaprenyl phosphate by sequential transfer of monosaccharides from nucleotide sugars by specific glycosyltransferases. Membrane-associated and -assembled repeating-unit oligosaccharides are thought to be translocated across the cytoplasmic membrane and polymerized to polysaccharides by a dedicated transport and polymerization machinery $[9,10]$. The overall capacity for this type of polysaccharide synthesis is encoded by clusters of genes that are readily detectable by their conserved structural composition (Wzy-dependent polymer gene cluster) and are commonly transcribed as a single transcript [11], although exceptions have been reported [12]. These clusters are found in many Gram-positive bacteria and are probably best documented for Streptococcus pneumonia [13,14]. The first genes in these clusters $(w z d, w z e, w z h)$ are involved in modulation of capsule synthesis and form a tyrosine phosphoregulatory circuit that controls polymer production [14]. These regulatory gene-cassettes are commonly followed by a variable number of genes encoding glycosyltransferases, which are involved in repeat unit synthesis on the cytoplasmic face of the cell membrane [14]. Once synthesized, the repeat unit is flipped across the membrane by a dedicated flippase (Wzx) and subsequently polymerized (Wzy) to form the extracellular polysaccharide [14].

EPS produced by LAB has received a lot of attention based on their role as thickening agents that are naturally produced during fermentation and influence the textural properties of fermented dairy and non-dairy products [9,15-18]. Besides the industrial relevance of polysaccharides produced by LAB in product characteristics, they may also play a role in the interaction between microbes and the host intestinal mucosa. While in pathogenic bacteria such as Streptococcus pneumoniae, polysaccharide capsules are extensively studied and were shown to play important roles in virulence by inhibiting opsonization and phagocytosis and are employed frequently as important serotyping antigens for epidemiological studies $[19,20]$, the role of cell surface polysaccharides of commensal or probiotic bacteria is far less understood. In Lactobacillus rhamnosus GG, biosynthesis of the high-molecular-weight, galactose-rich surface polysaccharide molecules negatively affects the bacterial capacity to bind to intestinal epithelial cells, which may be due to the shielding of adhesins on the bacterial cell surface [21]. Additionally, surface polysaccharides may also contribute to protection against intestinal innate immune factors such as the antimicrobial peptide LL-37 [22]. A direct role in host signaling has been proposed for purified surface polysaccharides of Lactobacillus casei Shirota that were shown to mediate the suppression of pro- inflammatory responses in macrophages [23]. Moreover, several polysaccharide biosynthesis-related genes of Lactobacillus plantarum were up-regulated in vivo in the GI-tract of mice and humans [24], and a recent study tentatively correlated elevated expression of cps genes to enhanced survival under GItract mimicking conditions in this species (van Bokhorst-van de Veen, unpublished observations). 
Here, we characterize the 4 cps gene clusters encoded by L. plantarum WCFS1, an extensively studied model organism for probiotic lactobacilli, aiming to identify bacterial molecules involved in microbe-host interactions with relevance for probiotic functions [25]. We constructed various $c p s$-cluster deficient strains, including single $(\Delta c p s 1 A-I, \Delta c p s 2 A-J, \Delta c p s 3 A-J, \Delta c p s 4 A-J)$, triple $(\triangle c p s 1 A-3 J)$, and quadruple $(\Delta c p s 1 A-3 J, \Delta c p s 4 A-J)$ deletion mutants. The consequences of cps cluster mutation were studied at the level of whole genome transcriptome profiles, and by determining the amount, molar mass and chemical composition of the surface polysaccharide fraction of the mutants in comparison to the wild-type strain. Finally, the impact of the cps cluster mutations on toll-like receptor (TLR)-dependent human nuclear factor (NF)- $\mathrm{kB}$ activation in host cells was evaluated using a TLR2 reporter cell line.

\section{Results and discussion}

\section{CPS biosynthesis cluster organization in L. plantarum WCFS1}

The L. plantarum WCFS1 genome contains two regions with surface-associated polysaccharide biosynthesis genes that are designated cps genes (Figure 1A and B). One region of $49 \mathrm{~kb}$ contains three gene clusters separated by transposase genes and has been identified as a genomic life-style island with high variability between L. plantarum strains [26]. Of these three gene clusters, cps 1 and cps 2 are identified only in strain WCFS1 and not in the other completely sequenced strains of the same species, while gene cluster cps3 is conserved in ST-III and ATCC 14917 but not in JDM1 (Figure 1; Additional file 1). A second region of $14 \mathrm{~kb}$ comprises the WCFS1 cps4 gene cluster and is conserved in other L. plantarum strains (Additional file 1). Clusters 2 and 4 have a typical structure of a Wzy-dependent polymer gene cluster (Figure 1; Additional file 1). The first three genes (cps $2 A B C$ and cps $4 A B C$ ) are homologous to the typical components of the tyrosine kinase phosphoregulatory circuit involved in control of capsule synthesis [14]. The fourth gene in both gene clusters is predicted to encode an UDP- $N$-acetylglucosamine 4-epimerase catalyzing the interconversion between UDP- $N$-acetyl-D-glucosamine and UDP- $N$-acetyl-Dgalactosamine (cps $2 D$, and cps $4 D)$. The fifth gene is predicted to encode the priming glycosyltransferase catalyzing the transfer of a sugar-1-phosphate from a UDP-sugar to the undecaprenyl-phosphate, the first step in the synthesis of the repeat unit (cps2E and cps4E). The cps clusters 2 and 4 contain three additional genes

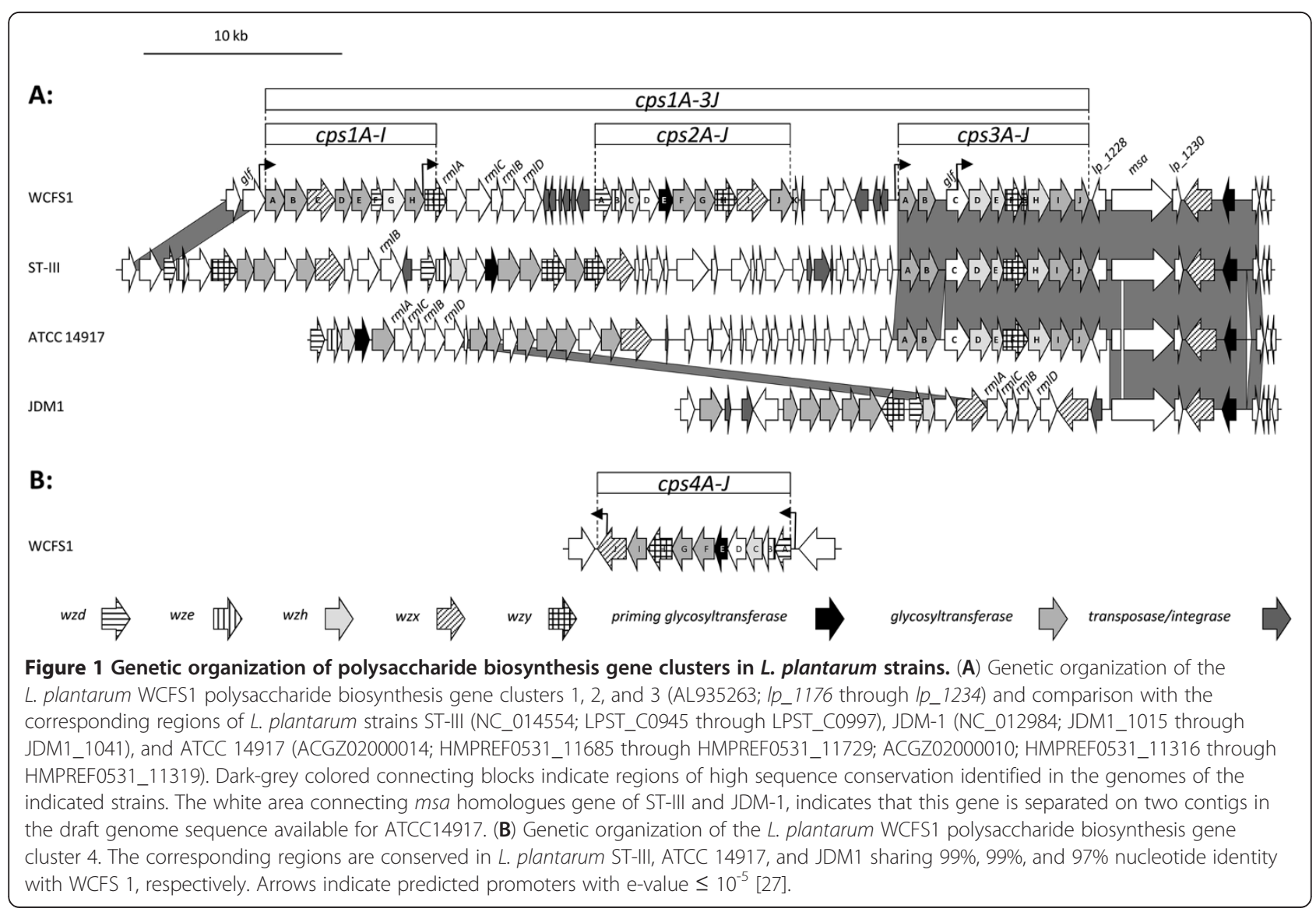


with high sequence similarity to glycosyltransferase genes (cps2FGJ and cps4FGI), indicating that the encoded polysaccharides would be made up of quatro-saccharide repeat units. The 3' regions of both clusters encode homologues of the typical flippase (cps2I and cps4J) and polymerase (cps2H and cps4H) functions required for capsule synthesis [14].

The organization of clusters 1 and 3 is different from that of clusters 2 and 4 . Cluster 3 appears to lack clear homologues of the chain-length modulator genes $w z d$, $w z e$, and wzh. However, it is tempting to speculate that cps3D, cps3E, and cps3H serve such function, as their gene products show low sequence similarities to several polysaccharide biosynthesis proteins of unknown functions and Cps3E shares distant homology (26\% identity) with the N-terminus of Clavibacter michiganensis subsp. michiganensis NCPPB 382 Wzc tyrosine kinase (functional homologues $w z d$ and $w z e$; NCBI accession number YP_001221462). The cps3 wzy homologue is split in two by a frame-shift caused by a single nucleotide insertion immediately upstream of the cps3F stop codon. It is unclear if a functional Wzy protein can be composed of Cps3F and Cps3G. The cluster contains three predicted glycosyltransferase genes but no priming glycosyltransferase gene. The presence of an acetyltransferase gene indicates that acetylation of the repeat units might take place. A set of genes encoding two transcriptional regulators and a mannose-specific adhesin protein separates the cps 3 gene cluster from a polysaccharide polymeraselike ( $\left.l p \_1231\right)$ and priming glycosyltransferase ( $\left.l p \_1233\right)$ gene (Figure 1). These genes could complete the polysaccharide synthesis machinery of cps3, which would then be predicted to be involved in the synthesis of a polysaccharide made up of acetylated quatro-saccharide repeat units. Notably, in Lactobacillus rhamnosus, exopolysaccharide gene clusters the priming glycosyltransferase genes are also separated from the body of the polysaccharide gene cluster [12].

The glf gene of cps cluster 1 is predicted to encode an UDP-galactopyranose mutase catalyzing the interconversion of UDP-galactopyranose and UDP-galacto1,4-furanose. The $g l f$ gene encodes a protein with $86 \%$ identity to the protein encoded by the cluster $3 \mathrm{glf}$ gene. The cps 1 cluster contains 5 predicted glycosyltransferase genes but like the cps3 cluster, it appears to lack a priming glycosyltransferase gene. In addition, it contains a predicted acetyltransferase gene, $w z x, w z y$, and $w z d$ homologues but no wze and wzh homologues. Expression of the cps 1 cluster is predicted to produce a polysaccharide comprising an acetylated hexa-saccharide repeat unit.

A functional role of the cps 1 and 3 clusters in production of surface glycans in L. plantarum WCFS1 is supported by the impact of individual deletion of either the cps1 or cps3 genecluster on the surface glycan characteristics (see below). Notably, the lack of a predicted priming glycosyltransferase and in both the cps3 and cps 1 clusters could imply that the glycosyltransferases encoded by these clusters interact with the undecaprenylphosphate-sugars derived from other pathways (e.g., cps clusters 2 and 4, or more remotely encoded priming glycosyltransferases like the lp_1233encoded function). However, an additional or alternative role of the cps geneclusters 1 and 3 in glycosylation of other surface molecules like teichoic acids and/or proteins as has been hypothesized before [2] can't be excluded at this stage.

\section{Deletion of cps clusters impacts on the L. plantarum transcriptome}

We previously generated global growth phase-dependent gene expression profiles of L. plantarum WCFS1 ranging from mid-logarithmic to late stationary growth phase (Daniela M. Remus, Fabrizia Fusetti, Jurgen Karczewski, Roger S. Bongers, Irene Konings, Bert Poolman, Maria L. Marco, Paul de Vos, Jerry M. Wells, Michiel Wels, Peter A. Bron and Michiel Kleerebezem, unpublished observations). Analysis of the $c p s$ cluster-related gene expression patterns revealed that the cps $1 A-I$ cluster-associated genes were highly expressed under laboratory conditions, whereas the genes of cps clusters $2 A-K, 3 A-I$ and $4 A-J$ were expressed at much lower levels (Figure 2).

Genome-wide transcriptional profiles of the single cps cluster deletion strains were generated, confirming the complete abolishment of expression of the genes affected by the mutation, and thus establishing the integrity of the mutants. These transcriptome analyses also revealed that cps cluster deletion affected the expression of variable gene sets in the individual cps cluster mutants as compared to the wild-type strain. Deletion of cps cluster 1 or 2 affected the largest group of genes (Table 1), whereas the transcriptome impact of deletion of cluster 4 was considerably smaller, and deletion of cluster 3 appeared the smallest (Table 1). Intriguingly, the transcriptome changes elicited upon deletion of cps clusters 1 or 2 as compared to the wild-type strain, shared a substantial number of downregulated genes $(87$ shared repressed genes) for which the encoded functions are associated with transport and metabolism of certain amino acids, including serine, glutamate, aspartate, asparagines, methionine, cysteine, and the ABCtransporter for the branch chain amino acids (Additional file 2). Notably, genes that were consistently upregulated upon deletion of cps clusters 1 and 2 (30 upregulated genes shared, Additional file 2) included some that encoded amino acid related functions, including a diand tri-peptide transport system as well as specific 


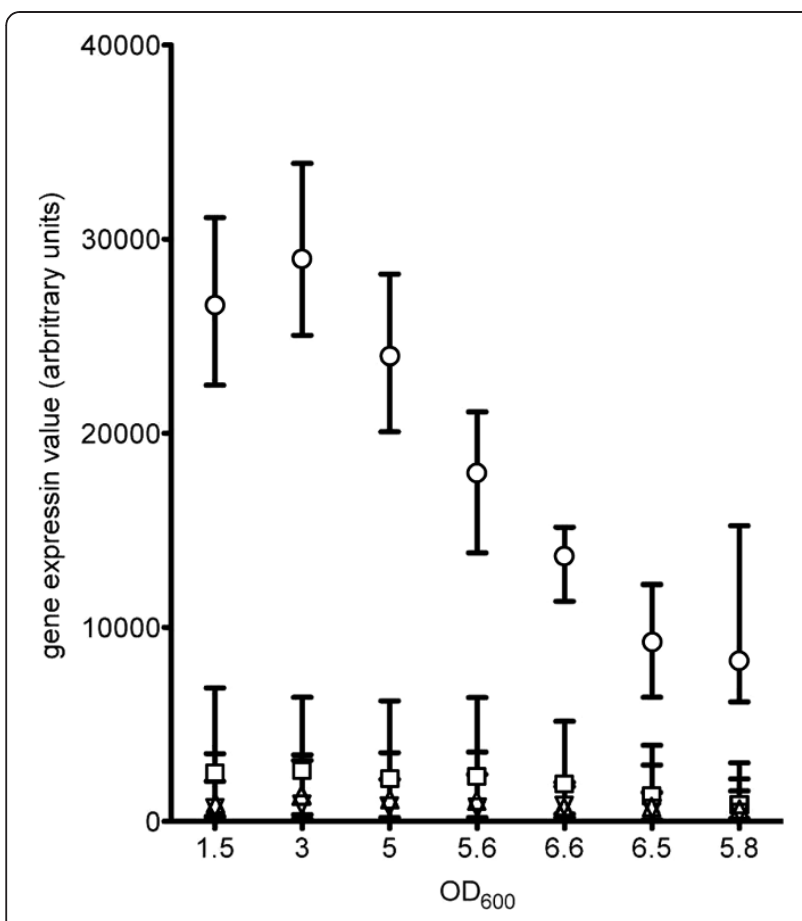

Figure 2 Growth phase dependent gene expression levels of individual $c p s$ gene clusters in L. plantarum WCFS1. Data is represented as average expression of cps genes of cluster 1 (circles), 2 (squares), 3 (upward pointing arrowheads) and 4(downward pointing arrowheads). Error bars represent the range of expression between the highest and lowest expressed gene in the different clusters.

glutamine and a facilitator-family branched-chain amino acid transport systems, but also several genes with predicted cell-envelope associated functions like several extracellular proteins, and genes involved in aminosugar synthesis and teichoic acid decoration. Remarkably, of the 20 upregulated genes observed in the cps cluster 3 deletion mutant relative to the wild-type strain, 11 were shared with the upregulated gene set found in the cps cluster 4 deletion mutant (Additional file 2). These genes included those encoding metabolic functions associated with pyruvate discipation or its control like, L-lactate dehydrogenase (ldhL2), pyruvate dehydrogenase ( $p d h A, p d h B$; also activated in the cps 2 deletion strain), NADH oxidase (nox5), but also several carbohydrate degradation and transport functions like an $\alpha$-amylase (malS), and the genetically linked and correlated transport functions for maltodextrins ( $m d x G)$, as well as two 6-phospho-beta-glucosidases (pbg5, and pbg4). Among these 6-phospho-beta-glucosidase encoding genes, the $p g b 4$ gene appeared to be induced in all cps cluster deletion strains, a feature shared by only two additional genes that appeared to be consistently downregulated in all mutants, and encode a prephenate dehydrogenase $(\operatorname{tyr} A)$, and a sodium-coupled N-acetylneuramidate transporter ( $\left.l p \_3563\right)$. In addition, expression of a FAD/FMN-containing dehydrogenase encoding gene ( $\left.l p \_0291\right)$ appeared to be differentially affected in the 4 cps deletion strains, i.e., downregulated upon deletion of cps cluster 1 or 2 , but upregulated when cps cluster 3 or 4 is deleted. Importantly, compensatory activation of one of the alternative cps clusters was not observed in any of the cps cluster deletion mutants. These results show that especially deletion of cps cluster 1 or 2 elicited pleiotropic transcriptome consequences, which appeared to be centered on genes with functions associated with metabolism and transport of various amino acids, but also included genes encoding several other functions. The observation that deletion of the capacity to produce CPS affected the expression of genes encoding specific transport and metabolism functions, may suggest that the presence of polysaccharides in the cell envelope plays a role in the access that the bacteria have to nutrients from the environment. Polysaccharides may function as macromolecules that sequester nutrients and thereby facilitate their import $[28,29]$, or alternatively they may form a capsular structure that surrounds the cell and retards or inhibits diffusion or transport of nutrients towards the membrane surface and thereby reduces trans-membrane transport efficiencies.

Impact of individual cps cluster mutations on L. plantarum surface glycan characteristics

Surface polysaccharides of L. plantarum WCFS1 and the cps cluster deletion mutants were isolated to assess their monosaccharide composition (Table 2). The results clearly demonstrated that the wild-type strain produces significant amounts of surface polysaccharides. Deletion of cluster 1 did not impact on the total amount of surface polysaccharides produced, which is remarkable in

Table 1 Number of genes, which were significantly changed in response to individual cps cluster deletions (false discovery rate adjusted $p$-value $\leq 0.05$ )

\begin{tabular}{|c|c|c|c|c|}
\hline & \multicolumn{4}{|c|}{ L. plantarum cps mutant strains } \\
\hline & $\mathrm{NZ3548cm} \Delta$ cps1A-I & NZ5333ACm $\Delta c p s 2 A-J$ & NZ3549Cm $\Delta c p s 3 A-J$ & NZ3534Cm $\Delta$ cps4A-J \\
\hline Upregulated & 62 & 84 & 20 & 58 \\
\hline Downregulated & 124 & 158 & 20 & 49 \\
\hline Total & 186 & 242 & 40 & 107 \\
\hline
\end{tabular}


Table 2 Surface glycan composition of L. plantarum WCFS1 and its cps cluster deletion mutant derivatives

\begin{tabular}{|c|c|c|c|c|c|c|}
\hline Sugar (\% of total sugars) & & & & lantarum str & & \\
\hline & WCSF1 & $\begin{array}{l}\mathrm{NZ} 3548 \mathrm{Cm} \\
\Delta \operatorname{cps} 1 \mathrm{~A}-\mathrm{I}\end{array}$ & $\begin{array}{l}\mathrm{NZ} 5333 \mathrm{ACm} \\
\Delta \text { cps2A-J }\end{array}$ & $\begin{array}{l}\mathrm{NZ3549Cm} \\
\Delta \text { cps3A-J }\end{array}$ & $\begin{array}{l}\mathrm{NZ3534Cm} \\
\Delta \text { cps4A-J }\end{array}$ & $\begin{array}{c}\mathrm{NZ3680Cm \Delta cps1A-3J}, \\
\Delta c p s 4 A-J\end{array}$ \\
\hline Rhamnose & 5.40 & n.d.* & 3.08 & 3.81 & 4.29 & n.d.* \\
\hline Glucosamine & 3.28 & 5.04 & 3.28 & 3.45 & 2.95 & 3.93 \\
\hline Galactose & 17.39 & 0.86 & 8.96 & 15.46 & 14.82 & 1.09 \\
\hline Glucose & 27.98 & 23.81 & 29.16 & 25.28 & 27.03 & 22.22 \\
\hline Galacturonic-acid & 45.66 & 69.79 & 55.52 & 52.00 & 50.92 & 72.23 \\
\hline Molar mass (kg/mol) & 28.93 & 16.5 & 29.36 & 27.79 & 26.82 & 17.64 \\
\hline $\begin{array}{l}\text { Polysaccharides isolated } \\
(\mathrm{mg} / \mathrm{L})\end{array}$ & 31.52 & 30.62 & 11.98 & 15.68 & 12.88 & 3.82 \\
\hline
\end{tabular}

${ }^{*}$ n.d. stands for 'not-detected'.

the light of the relatively high expression of the cps $1 A-I$ gene cluster in the wild-type (Figure 2) and the lack of compensatory expression changes of clusters 2 , 3, or 4 in the cps1A-I mutant. However, the deletion of cluster 1 led to a decreased molar mass of the isolated polysaccharides and influenced the monosaccharide composition, i.e. led to a reduced relative amount of galactose and to a complete lack of rhamnose. This observation establishes that the cps 1 cluster is functional and leads to production of a specific polysaccharide in L. plantarum WCFS1. In addition, the lack of rhamnose in the surface polysaccharide fraction obtained for strain NZ3548Cm $(\Delta c p s 1 A-I)$ is in agreement with the annotation of $\operatorname{cps} 1 H$ as the only rhamnosyltransferase found in all cps clusters. As the expression of the $r f b$ genes (genetically linked to the cps1 cluster) was not abolished in response to cps1A-I deletion (even slightly increased expression), it is likely that indeed the deletion of cps $1 H$ has led to the loss of rhamnose in the surface polysaccharide fraction.

Despite the low expression levels of clusters 2, 3 and 4 (Figure 2), deletion of each cluster reduced the production of surface polysaccharides, indicating their respective contributions to the overall surface polysaccharides produced by the wild-type strain (Table 2). The polysaccharides isolated from these mutants had a similar molecular mass as the polysaccharides isolated from the wild-type strain, indicating a particular role of the cps cluster 1 in polysaccharide chain length determination. Deletion of cluster 2 reduced the relative abundance of galactose in the surface polysaccharides, indicating the presence of this sugar in the cps2-encoded polysaccharide. In contrast, deletion of clusters 3 or 4 did not significantly affect the monosaccharide compositions of the polysaccharides produced. Notably, all polysaccharide molecules detected in the cps $2 A-J, \quad c p s 3 A-J$ and cps $4 A-J$ mutant strains contained rhamnose, whereby the presence of this monosaccharide in the surface polysaccharide fraction appears to be exclusively dependent on the cps1A-I cluster.

Deletion of all 4 cps clusters $(\triangle c p s 1 A-3 J, \Delta c p s 4 A-J)$ led to a substantial reduction (approximately $90 \%$ reduction in comparison to the wild-type) of the overall amount of surface polysaccharides isolated, which completely lacked rhamnose and showed a reduced relative amount of galactose, analogous to the $\Delta c p s 1 A-I$ strain. As this mutant is expected to lack the genetic capacity to produce these typical $w z y$-dependent polysaccharides, the surface glycans isolated from this strain may derive from other surface polymers that are synthesized via other mechanisms. These remaining polysaccharides contain glucose, glucosamine, and galacturonic acid and might derive from teichoic acids, glycosylated proteins, and/or peptidoglycan. Remarkably, mutation of the individual cps clusters did not lead to an apparent phenotypic change in terms of growth or cell morphology, while the deletion of all four clusters ( $\triangle$ cps $1 A-3 J, \Delta c p s 4 A-J)$ caused aggregation of cells and rapid sedimentation (data not shown), which may be explained by increased cell surface-hydrophobicity due to reduced amounts of the typically hydrophilic surface polysaccharides [30].

\section{Deletion of $c p s$ clusters influences $L$. plantarum TLR2-mediated NF-KB activation}

The effects of cps cluster deletion on host cell signaling were examined in HEK-293 reporter cell lines stably expressing human Toll-like receptor (TLR)2, which harbor a reporter plasmid containing firefly luciferase under the control of the human nuclear factor (NF) $-\kappa B$ promoter. HEK-293 cells do not normally produce TLRs and have been previously shown to be unresponsive to several microbial-associated molecular patterns (MAMPs) [31,32] HEK-293 cells transiently transfected with only the NF- $\mathrm{kB}$ reporter plasmid (pNiFTY) were unresponsive to TLR2 agonists of L. plantarum demonstrating the requirement of human TLR2 in this signaling pathway (data not shown). An $L$ plantarum 
wild-type like derivative (NZ3400Cm) activated TLR2dependent NF- $\mathrm{B}$ (Figure 3). Overall, activation of NF$\kappa \mathrm{B}$ remained unaffected by $\triangle c p s 1 A-I$ and $\triangle c p s 3 A-J$ but was slightly increased after stimulation by the $\Delta c p s 2 A-J$ and $\triangle c p s 4 A-J$ mutants, which are the strains that displayed the largest reduction in the amount of surface polysaccharides produced. Notably, exposure of TLR2expressing HEK-293 cells to $\Delta c p s 1 A-3 J$ and $\Delta c p s 1 A-3 J$, $\triangle c p s 4 A-J$ elicited the strongest responses and clearly activated NF-kB. Although cps4A-J appears to be expressed at very low levels, its deletion in the $\triangle c p s 1 A-3 J$ background led to a substantial increase of TLR2-mediated NF- $\mathrm{KB}$ activity of the resulting strain, demonstrating that deletion of all cps clusters and the concomitant severe reduction of surface polysaccharide production leads to enhanced release and/or exposure of TLR2-agonists. These results suggest a role of surface polysaccharides in the shielding of other L. plantarum cell envelope MAMPs such as lipoproteins and teichoic acids that could activate TLR signaling, as was previously proposed for Lactobacillus rhamnosus GG [21].

\section{Conclusions}

In L. plantarum, surface polysaccharide biosynthesis is encoded by 4 gene clusters, which independently contribute to the overall surface polysaccharides produced in this bacterium. Transcriptome analysis revealed that cps cluster deletion affected different sets of genes in the individual cps cluster mutants, whereas substantial numbers of regulated genes were shared between cps cluster mutant 1 and 2. Surface polysaccharide analysis revealed

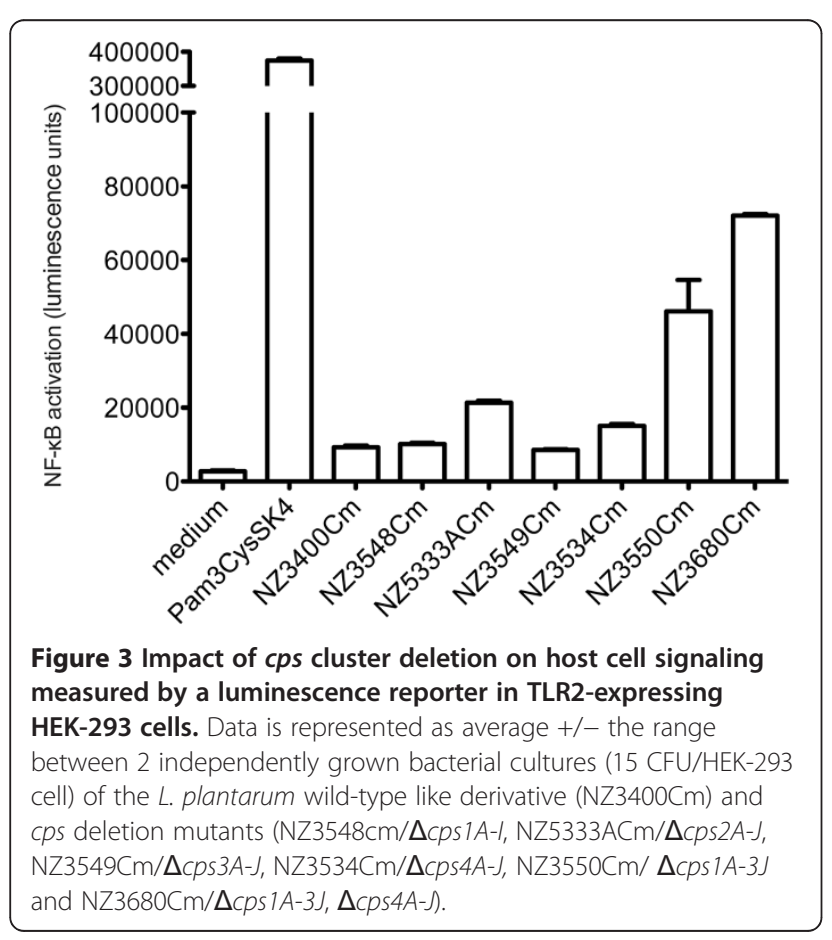

that the individual clusters influenced specific features of the polysaccharides produced, i.e. the amount, composition and molar mass. Although some of the individual mutants moderately affected TLR2-mediated NF- $\mathrm{BB}$ signaling, deletion of all clusters elicited a drastically increased NF- $\mathrm{kB}$ activation. In conclusion, the cps cluster encoded surface polysaccharides contribute to the L. plantarum cell surface architecture, and probably reduce release and/or exposure of TLR2-activating bacterial molecules.

\section{Bacterial culture conditions}

Escherichia coli strain TOP-10 (Invitrogen, Carlsbad, USA), which was used as intermediate cloning host, was grown aerobically in TY medium. L. plantarum WCFS1 [33,34] and its derivatives (Additional file 3) were either cultivated in Mann-Rogosa Sharpe (MRS; Merck, Darmstadt, Germany) or in 2-fold concentrated $(2 \times)$ chemical defined media (CDM) [35] supplemented with $1.5 \%$ (wt/vol) glucose without agitation. All bacteria were grown at $37^{\circ} \mathrm{C}$ and when appropriate, the media were supplemented with antibiotics; for E. coli and L. plantarum chloramphenicol was added at $10 \mu \mathrm{g} / \mathrm{mL}$, and during replica plating of L. plantarum chloramphenicol and erythromycin were added at final concentrations of $10 \mu \mathrm{g} / \mathrm{mL}$ and $30 \mu \mathrm{g} / \mathrm{mL}$, respectively.

\section{DNA manipulation techniques}

Plasmid DNA was isolated from E. coli using Jetstar columns following the manufacturer's instructions (Genomed $\mathrm{GmbH}$, Bad Oberhausen, Germany). Restriction endonucleases (Fermentas, St. Leon-Rot, Germany), KOD-DNA polymerase (Toyobo, Osaka, Japan) and T4 DNA ligase (Invitrogen, Carlsbad CA, USA) were used as recommended by the manufacturers. Primers were obtained from Sigma Aldrich (Zwijndrecht, The Netherlands), and DNA sequencing reactions were performed at BaseClear (Leiden, The Netherlands). Chromosomal DNA isolation, preparation of electrocompetent cells and DNA transformation of $L$. plantarum were performed as described previously [36,37].

\section{Construction of cps cluster deletion mutants}

Plasmids, primers and strains used in this study are listed in Additional file 3. The cps deletion mutants were constructed according to previously described methods [38], by which the target cps clusters were replaced by a chloramphenicol acetyltransferase (cat) gene cassette. In this study a derivative of the commonly used mutagenesis vector pNZ5319 [38], designated pNZ5319TAG (Bron et al., unpublished observations) was used that introduces a unique DNA-tag into the chromosome during gene deletion, which can be used for detection 
purposes (Bron et al., unpublished observations). The 5'- and 3'- flanking regions of the individual cps gene clusters (cps1A-I, cps2A-J, cps3A-J, and $\Delta$ cps4A-J) and of the cps1A-3J- and the $\mathrm{H}$-locus- spanning regions were amplified by PCR. The amplicons representing the flanking regions of the target cps clusters and the $\mathrm{H}$-locus were subsequently joined by a second PCR to the taglox66-F3/tag-lox71-R3 or tag-lox66-F2/tag-lox71-catR2 cassette, respectively. The resulting amplicons were cloned into SwaI-Ecl136II digested pNZ5319TAG. The obtained mutagenesis plasmids were integrated into the L. plantarum WCFS1 chromosome by double cross over replacement of the target gene (clusters) by the cat cassette, yielding the deletion mutant strains NZ3548Cm ( $\triangle c p s 1 A-I)$, NZ5333ACm ( $\Delta c p s 2 A-J)$, $\mathrm{NZ3549Cm}(\Delta c p s 3 A-J)$, and NZ3550Cm ( $\Delta c p s 1 A-3)$ ), as well as the tagged strain $\mathrm{NZ3400Cm}\left(l_{p} 2681-\mathrm{P}_{32}\right.$-catlp_2683). For construction of the quadruple mutant ( $\triangle$ cps1A-3I, $\triangle$ cps4A-J), the mutagenesis plasmid pNZ3550 (Additional file 3) was integrated into the $\triangle c p s 4 A-J$ chromosome, in which the cat cassette was removed by the temporal expression of the Cre recombinase [38], yielding the deletion mutant strain NZ3534Cm ( $\triangle$ cps1A-3I, $\triangle$ cps4A-J) [39]. The anticipated genotype of all mutants was confirmed by PCR using primers flanking the sites of recombination.

\section{RNA isolation}

L. plantarum and its cps-cluster deficient derivatives were grown in $2 \times \mathrm{CDM}$ and RNA was isolated according to previous described methods $[40,41]$. In short, following methanol quenching [42], cells were harvested by centrifugation $\left(6000 \times \mathrm{g}, 20 \mathrm{~min}, 4^{\circ} \mathrm{C}\right)$, resuspended in $400 \mu \mathrm{L}$ ice-cold CDM medium and transferred to tubes containing $500 \mu \mathrm{L}$ phenol/chloroform solution (4:1 [v/v]), $30 \mu \mathrm{L} 10 \%$ sodium dodecyl sulfate, $30 \mu \mathrm{L} 3 \mathrm{M}$ sodium acetate ( $\mathrm{pH} 5.2$ ), and $0.5 \mathrm{~g}$ zirconium beads. Cells were disrupted by bead beating using a Savant FastPrep FP120 instrument (Qbiogen Inc., Illkirch, France), and RNA was purified from the aqueous phase using the High Pure Isolation Kit (Roche Diagnostics, Germany). RNA concentration and purity were determined using A260 and A280 measurements using a ND-1000 spectrometer (NanoDrop Technologies Inc., Wilmington, United States), and RNA quality was verified with a 2100 Bioanalyzer (Agilent Technologies, Amstelveen, the Netherlands). Samples that displayed a 23S/16S RNA ratio equal or superior to 1.6 were used for labeling.

\section{Transcriptome analysis and interpretation}

$3 \mu \mathrm{g}$ RNA was used for cDNA synthesis. Cyanine-3 (Cy3) and cyanine-5 (Cy5) cDNA labeling was performed as described previously [43], using the CyScribe Post-Labeling and Purification kits according to the manufacturer's instructions (Amsersham Biosciences, Buckinghamshire, UK). Cy-dye-labeled cDNAs $(0.5 \mu \mathrm{g}$ each) were hybridized to L. plantarum WCFS1 printedoligonucleotide DNA microarrays (Agilent Technologies, Amstelveen, the Netherlands). The array design and transcriptome data were deposited under platform GPL13984 and accession number GSE34690 in NCBI's Gene Expression Omnibus (GEO) [44,45] at http://www. ncbi.nlm.nih.gov/geo/. Hybridization and scanning procedures were performed as previously described [43]. Slide scanning was carried out at several photo multiplier tube (pmt) values, and the optimal scan of each individual microarray was selected on the basis of signal distribution (combination of a low number of saturated spots and a low number of low signal spots). The data were normalized using the Lowess normalization as available in MicroPrep [46]. For statistical significance, Benjamini and Hochberg's False Discovery Rate (FDR) was used [47], with a FDR-adjusted p-value cutoff of 0.05 , employed for genes showing at least 2-fold altered expression.

\section{Surface polysaccharide isolation and determination of glycan composition}

Surface polysaccharides were isolated and characterized according to previously described methods [48,49]. L. plantarum WCFS1 and its mutant derivatives were grown in $2 \times \mathrm{CDM}$ until late stationary phase. After growth, cultures were incubated at $55^{\circ} \mathrm{C}$ for $1 \mathrm{~h}$, followed by pelleting of the bacterial cells (6000 $\mathrm{x} \mathrm{g}$, $15 \mathrm{~min}$, room temperature). The supernatants were supplemented with erythromycin $(30 \mu \mathrm{g} / \mathrm{mL})$, transferred to dialysis tubes (molecular weight cutoff of 12-14000 Da, Fisher Scientific, Landsmeer, The Netherland) and dialyzed overnight against running tap water followed by dialysis for $4 \mathrm{~h}$ against deionized water. The dialyzed samples were freeze-dried and stored at $-20^{\circ} \mathrm{C}$ until further analysis.

Samples were dissolved in eluent $\left(100 \mathrm{mM} \mathrm{NaNO}_{3}+\right.$ $0.02 \% \mathrm{NaN}_{3}$ ), and polysaccharides were separated by size exclusion chromatography (SEC), light scattering was measured at $632.8 \mathrm{~nm}$, UV-adsorption of proteins was measured at $280 \mathrm{~nm}$, viscosity was measured with a viscosity detector (ViscoStar, Wyatt Technologies, Santa Barbara, USA), and sample concentrations were measured by determining their refraction index. During SEC, polysaccharide peaks were collected and hydrolyzed with $2 \mathrm{M}$ trifluoroacetic acid (TFA), dried and dissolved in water. The quantitative monosaccharide composition of the polysaccharide fractions was analyzed using High Performance Anion Exchange Chromatography with Pulsed Amperometric Detection (HPAEC-PAD) equipped with a gold electrode. The monosaccharides were eluted isocratically with $16 \mathrm{mM}$ 
sodium hydroxide followed by the elution of the acid monosaccharides starting at $20 \mathrm{~min}$ with a linear gradient to $200 \mathrm{mM}$ sodium hydroxide $+500 \mathrm{mM}$ sodium acetate in $20 \mathrm{~min}$. Data analysis was performed with Dionex Chromeleon software version 6.80. Quantitative analyses were carried out using standard solutions of the monosaccharides (rhamnose, galactosamine, glucosamine, galactose, glucose, mannose, xylose, galacturonic acid, and glucuronic acid) (Sigma-Aldrich, St. Louis, USA).

\section{Host cell signaling assay}

Human embryonic kidney (HEK)-293 cells not expressing TLR receptors but harbouring pNIFTY, а NF- $\mathrm{kB}$ luciferase reporter construct (Invivogen, Toulouse, France) were used as the negative control in the NF- $\mathrm{kB}$ assays. HEK-293 cells (Invivogen, Toulouse, France) expressing human TLR2 and pNIFTY, a NF-kB luciferase reporter construct (Invivogen) were derived as previously described [50]. The HEK-293 TLR2 reporter cell line was seeded at $5 \times 10^{5}$ cells $/ \mathrm{cm}^{2}$ in 96 -well plates and incubated overnight under standard culture conditions. Cells were then stimulated with 2 independently grown bacterial cultures of the $L$ plantarum wild-type like derivative $(\mathrm{NZ} 3400 \mathrm{Cm})$ or cps deletion mutants (15 CFU/HEK-293 cell). After this incubation period, the medium was replaced with Bright-Glo ${ }^{\mathrm{TM}}$ (Promega Benelux BV, Leiden, The Netherlands), the plate was vortexed, and the luminescence was measured using a Spectramax M5 (Molecular Devices, Sunnyvale, USA). As positive control, the TLR2 agonist Pam3CysSK4 $(5 \mu \mathrm{g} / \mathrm{mL})$ was used, and as negative control, no bacterial cells were added to the HEK-293 cells.

\section{Additional files}

Additional file 1: Shared regulated genes between cps cluster
mutant.
Additional file 2: CPS biosynthesis-related genes of L. plantarum
WCFS1 and their homologues in strains ATTC 14917, JDM1, and
ST-III.
Additional file 3: Primers, plasmids, and strains used in this study.

\section{Competing interests}

The authors declare that they have no competing interests.

\section{Authors' contributions}

DMR, RvK, PAB and MK drafted the manuscript. MK, JW and PAB supervised the project. DMR, IlvS, NT, and RSB performed the experiments, DMR and MK analyzed the data, RvK performed cps cluster analysis and comparison, MW designed the hybridization scheme of the microarray experiments and processed the transcriptome data. All authors read and approved the manuscript.

\section{Acknowledgements}

We gratefully acknowledge Roland Siezen and Bernadette Renckens for their expert assistance with the cps gene cluster annotations. The authors cordially thank Guido Staring (NIZO food research, Ede, the Netherlands) for performing HPAEC and acknowledge the managerial role of Dr. Paul de Vos (University Medical Centre Groningen, The Netherlands).

\section{Author details}

${ }^{1}$ TI Food \& Nutrition, Nieuwe Kanaal 9A, 6709 PA Wageningen, The Netherlands. ${ }^{2} \mathrm{NIZO}$ food research, Kernhemseweg 2, 6718 ZB Ede, The Netherlands. ${ }^{3}$ Laboratory for Microbiology, Wageningen University, Dreijenplein 10, 6703 HB Wageningen, The Netherlands. ${ }^{4}$ Purac, P.O. Box 20, 4200 AA Gorinchem, The Netherlands. ${ }^{5}$ Host-Microbe Interactomics Group, Wageningen University, De Elst 1, 6708 WD Wageningen, The Netherlands. ${ }^{6}$ Kluyver Centre for Genomics of Industrial Fermentation, P.O. Box 5057, 2600 GA Delft, The Netherlands.

Received: 28 August 2012 Accepted: 8 November 2012

Published: 21 November 2012

\section{References}

1. Silhavy TJ, Kahne D, Walker S: The bacterial cell envelope. Cold Spring Harb Perspect Biol 2010, 2:a000414.

2. Kleerebezem M, Hols P, Bernard E, Rolain T, Zhou MM, Siezen RJ, Bron PA: The extracellular biology of the lactobacilli. FEMS Microbiol Rev 2010, 34:199-230.

3. Smit G, Smit BA, Engels WJ: Flavour formation by lactic acid bacteria and biochemical flavour profiling of cheese products. FEMS Microbiol Rev 2005, 29:591-610.

4. FAO/WHO: Guidelines for the evaluation of probiotics in food. London, Ontario: Report at the Joint FAO/WHO Working Group on Drafting Guidelines for the Evaluation of Probiotics in Food; 2002.

5. van den Berg DJC, Smits A, Pot B, Ledeboer AM, Kersters K, Verbake JMA, Verrips $C T$ : Isolation, screening and identification of lactic acid bacteria from traditional food fermentation processes and culture collections. Food Biotechnol 1993, 7:189-205.

6. Ruas-Madiedo P, Hugenholtz J, Zoon P: An overview of the functionality of exopolysaccharides produced by lactic acid bacteria. Int Dairy J 2002, 12:163-171.

7. De Vuyst L, De Vin F, Vaningelgem F, Degeest B: Recent developments in the biosynthesis and applications of heteropolysaccharides from lactic acid bacteria. Int Dairy J 2001, 11:687-707

8. Ismail B, Nampoothiri KM: Production, purification and structural characterization of an exopolysaccharide produced by a probiotic Lactobacillus plantarum MTCC 9510. Arch Microbiol 2010, 192:1049-1057.

9. De Vuyst L, Degeest B: Heteropolysaccharides from lactic acid bacteria. FEMS Microbiol Rev 1999, 23:153-177.

10. Delcour J, Ferain T, Deghorain M, Palumbo E, Hols P: The biosynthesis and functionality of the cell-wall of lactic acid bacteria. Antonie van Leeuwenhoek 1999, 76:159-184.

11. van Kranenburg R, Marugg JD, Van Swam II, Willem NJ, de Vos WM: Molecular characterization of the plasmid-encoded eps gene cluster essential for exopolysaccharide biosynthesis in Lactococcus lactis. Mol Microbiol 1997, 24:387-397.

12. Péant B, LaPointe G, Gilbert C, Atlan D, Ward P, Roy D: Comparative analysis of the exopolysaccharide biosynthesis gene clusters from four strains of Lactobacillus rhamnosus. Microbiology 2005, 151:1839-1851.

13. Bentley SD, Aanensen DM, Mavroidi A, Saunders D, Rabbinowitsch E, Collins M, Donohoe K, Harris D, Murphy L, Quail MA, et al: Genetic analysis of the capsular biosynthetic locus from all 90 pneumococcal serotypes. PLoS Genet 2006, 2:e31.

14. Yother J: Capsules of Streptococcus pneumoniae and other bacteria: paradigms for polysaccharide biosynthesis and regulation. Annu Rev Microbiol 2011, 65:563-581

15. Petry S, Furlan S, Waghorne E, Saulnier L, Cd J, Maguin E: Comparison of the thickening properties of four Lactobacillus delbrueckii subsp. bulgaricus strains and physicochemical characterization of their exopolysaccharides. FEMS Microbiol Lett 2003, 221:285-291.

16. Laws AP, Marshall VM: The relevance of exopolysaccharides to the rheological properties in milk fermented with ropy strains of lactic acid bacteria. Int Dairy J 2001, 11:709-722.

17. van Kranenburg R, Boels IC, Kleerebezem M, de Vos WM: Genetics and engineering of microbial exopolysaccharides for food: approaches for the production of existing and novel polysaccharides. Curr Opin Biotechnol 1999, 10:498-504. 
18. Di Cagno R, De Angelis M, Limitone A, Minervini F, Carnevali P, Corsetti A, Gaenzle M, Ciati R, Gobbetti M: Glucan and fructan production by sourdough Weissella cibaria and Lactobacillus plantarum. J Agric Food Chem 2006, 54:9873-9881.

19. García E, Llull D, Muñoz R, Mollerach M, López R: Current trends in capsular polysaccharide biosynthesis of Streptococcus pneumoniae. Res Microbiol 2000, 151:429-435.

20. Henrichsen J: Six newly recognized types of Streptococcus pneumoniae. J Clin Microbiol 1995, 33:2759-2762.

21. Lebeer S, Verhoeven TLA, Francius G, Schoofs G, Lambrichts I, Dufrene $Y$, Vanderleyden J, De Keersmaecker SCJ: Identification of a gene cluster for the biosynthesis of a long, galactose-rich exopolysaccharide in Lactobacillus rhamnosus GG and functional analysis of the priming glycosyltransferase. Appl Environ Microbiol 2009, 75:3554-3563.

22. Lebeer S, Claes IJJ, Verhoeven TLA, Vanderleyden J, De Keersmaecker SCJ: Exopolysaccharides of Lactobacillus rhamnosus GG form a protective shield against innate immune factors in the intestine. Microb Biotechnol 2011, 4:368-374

23. Yasuda E, Serata M, Sako T: Suppressive Effect on Activation of Macrophages by Lactobacillus casei Strain Shirota Genes Determining the Synthesis of Cell Wall-Associated Polysaccharides. Appl Environ Microbiol 2008, 74:4746-4755.

24. Marco ML, de Vries MC, Wels M, Molenaar D, Mangell P, Ahrne S, de Vos WM Vaughan EE, Kleerebezem M: Convergence in probiotic Lactobacillus gutadaptive responses in humans and mice. ISME J 2010, 4:1481-1484.

25. Remus DM, Kleerebezem M, Bron PA: An intimate tête-à-tête - How probiotic lactobacilli communicate with the host. Eur J Pharmacol 2011, 668:S33-42.

26. Siezen R, van Hylckama Vlieg JE: Genomic diversity and versatility of Lactobacillus plantarum, a natural metabolic engineer. Microb Cell Fact 2011, 10:\$3

27. Wels M, Kleerebezem M, Siezen RJ: Unraveling the regulatory network of Lactobacillus plantarum WCFS1. Wageningen, The Netherlands: Wageningen UR (University \& Research centre); 2008

28. McDougald D, Rice SA, Barraud N, Steinberg PD, Kjelleberg S: Should we stay or should we go: mechanisms and ecological consequences for biofilm dispersal. Nat Rev Microbiol 2011, 10:39-50.

29. Costerton JW, Lewandowski Z, Caldwell DE, Korber DR, Lappin-Scott HM: Microbial biofilms. Annu Rev Microbiol 1995, 49:711-745.

30. Davey ME, Duncan MJ: Enhanced biofilm formation and loss of capsule synthesis: deletion of a putative glycosyltransferase in Porphyromonas gingivalis. J Bacteriol 2006, 188:5510-5523.

31. Medvedev AE, Vogel SN: Overexpression of CD14, TLR4, and MD-2 in HEK 293T cells does not prevent induction of in vitro endotoxin tolerance. J Endotoxin Res 2003, 9:60-64

32. Latz E, Visintin A, Lien E, Fitzgerald KA, Monks BG, Kurt-Jones EA, Golenbock DT, Espevik T: Lipopolysaccharide rapidly traffics to and from the Golgi apparatus with the toll-like receptor 4-MD-2-CD14 complex in a process that is distinct from the initiation of signal transduction. J Biol Chem 2002, 277:47834-47843.

33. Kleerebezem M, Boekhorst J, van Kranenburg R, Molenaar D, Kuipers OP, Leer R, Tarchini R, Peters SA, Sandbrink HM, Fiers M, et al: Complete genome sequence of Lactobacillus plantarum WCFS1. Proc Natl Acad Sci US A 2003, 100:1990-1995.

34. Siezen RJ, Francke C, Renckens B, Boekhorst J, Wels M, Kleerebezem M, van Hijum SA: Complete resequencing and reannotation of the Lactobacillus plantarum WCFS1 genome. J Bacteriol 2012, 194:195-196.

35. Teusink B, van Enckevort FHJ, Francke C, Wiersma A, Wegkamp A, Smid EJ, Siezen RJ: In silico reconstruction of the metabolic pathways of Lactobacillus plantarum: Comparing predictions of nutrient requirements with those from growth experiments. Appl Environ Microbiol 2005, 71:7253-7262

36. Ferain T, Garmyn D, Bernard N, Hols P, Delcour J: Lactobacillus plantarum IdhL gene: overexpression and deletion. J Bacteriol 1994, 176:596-601.

37. Josson K, Scheirlinck T, Michiels F, Platteeuw C, Stanssens P, Joos H, Dhaese $P$, Zabeau M, Mahillon J: Characterization of a gram-positive broad-host-range plasmid isolated from Lactobacillus hilgardii. Plasmid 1989, 21:9-20.

38. Lambert JM, Bongers RS, Kleerebezem M: Cre-lox-based system for multiple gene deletions and selectable-marker removal in Lactobacillus plantarum. Appl Environ Microbiol 2007, 73:1126-1135.
39. Andre G, Deghorain M, Bron PA, van Swam II, Kleerebezem M, Hols P, Dufrene YF: Fluorescence and atomic force microscopy imaging of wall teichoic acids in Lactobacillus plantarum. ACS Chem Biol 2011, 6:366-376.

40. Saulnier DAA, Molenaar D, de Vos WA, Gibson GR, Kolida S: Identification of prebiotic fructooligosaccharide metabolism in Lactobacillus plantarum WCFS1 through microarrays. App/ Environ Microbiol 2007, 73:1753-1765.

41. Marco ML, Peters THF, Bongers RS, Molenaar D, van Hemert S, Sonnenburg $J$, Gordon I, Kleerebezem M: Lifestyle of Lactobacillus plantarum in the mouse caecum. Environ Microbiol 2009, 11:2747-2757.

42. Pieterse B, Jellema $\mathrm{RH}$, van der Werf MJ: Quenching of microbial samples for increased reliability of microarray data. J Microbiol Methods 2006, 64:207-216

43. Meijerink M, van Hemert S, Taverne N, Wels M, de Vos P, Bron PA, Savelkoul HF, van Bilsen J, Kleerebezem M, Wells JM: Identification of Genetic Loci in Lactobacillus plantarum That Modulate the Immune Response of Dendritic Cells Using Comparative Genome Hybridization. PLoS One 2010, 5:e10632.

44. Barrett T, Edgar R: Gene expression omnibus: Microarray data storage, submission, retrieval, and analysis. In DNA Microarrays, Part B: Databases and Statistics. Volume 411th edition.; 2006:352-369. Methods in Enzymology.

45. Edgar R, Domrachev M, Lash AE: Gene Expression Omnibus: NCBI gene expression and hybridization array data repository. Nucleic Acids Res 2002, 30:207-210.

46. van Hijum SA, Garcia De La Nava J, Trelles O, Kok J, Kuipers OP: MicroPreP. a cDNA microarray data pre-processing framework. Appl Bioinformatics 2003, 2:241-244.

47. Benjamini $Y$, Hochberg $Y$ : Controlling the false discovery rate: a practical and powerful approach to multiple testing. J R Stat Soc Series B Stat Methodol 1995, 57:289-300

48. Looijesteijn PJ, Hugenholtz J: Uncoupling of growth and exopolysaccharide production by Lactococcus lactis subsp. cremoris NIZO B40 and optimization of its synthesis. J Biosci Bioeng 1999, 88:178-182.

49. Looijesteijn PJ, Boels IC, Kleerebezem M, Hugenholtz J: Regulation of exopolysaccharide production by Lactococcus lactis subsp. cremoris by the sugar source. Appl Environ Microbiol 1999, 65:5003-5008.

50. Karczewski J, Troost FJ, Konings I, Dekker J, Kleerebezem M, Brummer RJM, Wells JM: Regulation of human epithelial tight junction proteins by Lactobacillus plantarum in vivo and protective effects on the epithelial barrier. Am J Physiol Gastrointest Liver Physiol 2010, 298:G851-859.

doi:10.1186/1475-2859-11-149

Cite this article as: Remus et al:: Impact of 4 Lactobacillus plantarum capsular polysaccharide clusters on surface glycan composition and host cell signaling. Microbial Cell Factories 2012 11:149.

\section{Submit your next manuscript to BioMed Central and take full advantage of:}

- Convenient online submission

- Thorough peer review

- No space constraints or color figure charges

- Immediate publication on acceptance

- Inclusion in PubMed, CAS, Scopus and Google Scholar

- Research which is freely available for redistribution 